\title{
Recent advances in energy efficient-QoS aware MAC protocols for wireless sensor networks
}

\author{
Bashir A Muzakkari ${ }^{1}$, Mohamad A Mohamed ${ }^{1 *}$, Mohd F A Kadir ${ }^{1}$, Zarina Mohamad ${ }^{1}$ and \\ Norziana Jamil ${ }^{2}$ \\ Faculty of Informatics \& Computing, Universiti Sultan Zainal Abidin, Besut Campus, Malaysia ${ }^{1}$ \\ Faculty of Institute of Informatics and Computing in Energy, Universiti Tenaga Nasional, Malaysia ${ }^{2}$
}

Received: 16-May-2018; Revised: 03-September-2018; Accepted: 06-September-2018

(C)2018 ACCENTS

\begin{abstract}
Wireless sensor networks (WSNs) is a distribution of several tiny, low-cost sensor nodes, wirelessly connected altogether for the purpose of monitoring physical or environmental conditions. Due to the vast interest for WSN, a rapid technological breakthrough has been observed in sensor elements such as processor, operating system, radio, and battery. From the perspective of seven layer approach, the medium access control (MAC) protocols are identified as the most crucial element, being responsible for coordinating communication amongst the sensor nodes. In addition, the functionality of the WSN MAC protocol has a subtle influence on parameters such as battery consumption, packet collision, network lifetime and latency. In this paper, we survey some of the most recent WSN contention-based, scheduling-based, and hybrid MAC protocols by focusing on their underlying principle, various advantages and limitations and their applications. Treating energy saving as the benchmark, further examining the directed towards the treatment of quality of service (QoS) performance metrics within these particular protocols. The result shows that the majority of the protocols leaned towards energy conservation with other parameters are either supported partially or traded off. Latency, throughput, bandwidth utilization, channel utilization is not considered in the design of most of the protocols. Indeed, the energy domain has gotten a vital breakthrough with the advent of other modes of energy saving such as energy harvesting techniques. However, other parameters such as latency, throughput, packet loss, network and bandwidth availability that comes under QoS metrics also play a critical role in future development of MAC protocols for WSNs.
\end{abstract}

\section{Keywords}

WSN, MAC protocol, Quality of service, Energy efficient, Energy consumption.

\section{Introduction}

The emergence of wireless technologies has facilitated immense opportunities for optimal and efficient communication system. WSN is an effective communication technique which has expanded the application space ranging from public utilities, industrial monitoring and control, and defense and military operations. In general, WSN plays a significant role in wireless communication tool, and it has a great potential to serve as the next generation communication technology and for the future ubiquitous network. Such robustness have widened the usability of WSNs into various major applications and as such the technological development is expanding rapidly across the scientific horizon.

*Author for correspondence

212
Interests were shown from the scientific society, academia, and industries to enhance the quality and performance of WSNs [1].

Due to lightweight characteristics, sensor node comes with many limitations such as radio coverage and processing power. However, the most fundamental, of which most of the protocols were designed for the energy efficiency. As the applications are becoming more complex such that found in healthcare, industrial monitoring, and military surveillance, the expectation has grown from a little energy efficient devices into the support for the QoS. QoS faces challenges with respect to the limited resources of its sensor nodes, unpredictable condition of wireless links, and severe environments for its operations. Both the energy consumption and QoS support are defined by the MAC protocol. However, most researchers concentrated largely on the WSN energy 
efficiency for the maximization of network lifetime while neglecting other parameters.

Some MAC protocols for WSNs were proposed in the literature [2-4], most of which to minimize energy consumption. Among the proposed protocols, only a few consider other performance metrics such as latency, throughput, reliability and some design concerns that may fulfil the QoS support requirements.

This paper investigates the treatment of QoS parameters into an energy-aware MAC protocols for WSNs. This paper differs from current survey papers in that it analyses recently proposed MAC protocols based on their suitability for not only energy efficient but also QoS support. We survey recent developments in energy-efficient and QoS based MAC protocols, and classify them based on their medium access schemes and the nature of their respective algorithms and design approaches.

This paper is organized as follows: Section 2 discusses the MAC, causes of energy waste and challenges of designing a suitable MAC protocol for WSNs followed by investigation into different categories of MAC protocols highlighting on their strengths and weaknesses. Section 3 presents a discussion and future research direction in the design of MAC protocols. Section 4 concludes the paper.

\section{Medium access control}

Seven main functionalities define a sensor node; these are initiation, sensing, actuating, logging, transient, processing, and communicating. From a hardware point of view, a sensor node is equipped with sensing and computing units, radio transceivers and power components [5]. Sensor communication can be represented by the infamous seven layers OSI approach. In layer 2, MAC protocol coordinates channel's access in a network, enabling an enormous number of nodes to share communication channels at the same time. From time to time, MAC protocol is responsible for resolving contentions between nodes to gain access to the channel.

Being a coordinator for communication among sensor nodes, the design choice for MAC protocol is very much dependent upon nodes' and networks' parameters such as energy utilization, packet transmission, network lifetime and latency. Since many issues are bound to the MAC layer, an efficient MAC protocol is the primary factor to be considered when designing any WSN application.
By and large, energy consumption is considered as the most fundamental issue of WSN, and it is widely affected by the communication functionality of sensor nodes. In this respect, we can map energy consumption to the seven layers of the OSI protocol stack. For the solution, energy conservation can be implemented in diverse layers of the TCP/IP protocol stack. However, the MAC layer is identified as the most effective considering its ability to directly control radio communication. Thus, to ensure the most energy efficient allocation and usability, and to prolong network lifetime, we are therefore in need of an improved MAC protocol which is energy efficient.

The performance of all upper layers depends on the MAC layer. Therefore, QoS support cannot be achieved at the network and transport layers without the conjecture of a MAC protocol that supports reliable communication and resolves the problems of medium sharing [6]. At this juncture, QoS provisioning at the MAC layer is of great importance to the overall QoS requirements in WSN.

Problems in MAC layer are caused by several attributes and trade-offs such as energy efficiency, collision avoidance, scalability and adaptability, channel utilization, fairness, and QoS support. The main parameters for QoS that of the utmost concerns are latency (the delay), jitter and packet loss. Other parameters such as reliability, network, and bandwidth availability are also belonged to QoS but having less priority.

\subsection{Causes of energy waste}

If we consider the whole communication stack, many challenges are waiting to be addressed in relation to energy wastage. Nevertheless, specific to the MAC layer, energy waste in WSN occurs as a result of reasons such as collision, idle listening, overhearing, control packet overhead, and over-emitting [2, 7]. Collision happens when a packet is corrupted during transmission and has to be dropped, energy consumption and latency increases as a result of the packet retransmission. In idle listening, a node listens to receive a packet that is not sent. Idle listening consumes half or all of the energy required for receiving. Overhearing occurs when a node received a transmitted packet that is meant for other nodes. Control packet overhead also consumes energy by sending and receiving control packets. Over-emitting is caused by message transmission to a node that is not ready. To tackle this issue, a good design of MAC protocol is needed. However, this shall not 
forget other issues that also must come under scrutiny.

\subsection{Characteristics of a good MAC design}

To achieve a good MAC protocol for WSNs, the following attributes should be given greater consideration [8] and be included in the design process.

Energy Efficiency: Sensor nodes are powered by battery, it is, therefore, impractical to recharge or replace their batteries. To extend the network lifetime, energy-efficient protocols must be defined to reduce potential energy wastes.

Latency: Latency requirement is determined by the WSN application used. In WSN, detected events are required to be relayed to the sink node in real-time for further action.

Throughput: It also varies with applications. Some WSN applications require sampling the information with reasonable sequential resolution, it is therefore required that the sink node should receive more data than a normal node.
Scalability \& Adaptability: The network must be able to accommodate changes in the network size, topology and node density that may occur as a result of node lifetime, newly deployed nodes and or node mobility. Other features such as fairness, throughput, latency, and channel utilization are regarded as ancillary in sensor networks [2].

Fairness: In numerous sensor applications with bandwidth constraints, it is very essential to guarantee that the sink node fairly receives information from all nodes. However, energy efficiency and throughput are the major aspects among all of the aforementioned aspects. Energy efficiency can be achieved by minimizing energy waste.

Channel Utilization: This reflects how the entire channel bandwidth is utilized during communication. Bandwidth is a valuable resource for wireless communication. So, the MAC protocols designed for WSN should maximize the utilization of this scarce resource. Numerous mechanisms have been put forward to address the major problems at MAC layer. Table 1 tries to catalogue the mapping of each mechanism against its respective issues.

Table 1 Mapping of MAC mechanisms for different MAC issues

\begin{tabular}{ll}
\hline Mechanisms & Issues addressed \\
\hline Duty cycling & Idle listening, collision, and overhearing \\
Adaptive duty cycling & Fixed duty cycling and collision \\
Adaptive sleeping and reusing of channel & Idle listening, overhearing, overhead and collision \\
Low power listening (LPL) & idle listening and overhead \\
Short preamble & Overhearing, idle listening, and overhead \\
Anycast and convergent packet forwarding & Overhead \\
On-demand prediction error correction & Collision and over-emitting \\
Clustering & Overhead, idle listening, Collision \\
Adaptive election algorithm & Collision \\
Slot assignment & Idle listening, overhearing, collision, and over-emitting \\
Channel polling & Collision \\
Sender-initiated & Collision \\
Receiver initiated & Idle listening, overhearing, and collision \\
Data prediction and more-to-send notification & Overhead \\
Node synchronization offset wake-up schedules & Collision, overhearing, and idle listening \\
Advertisement scheme & Collision, overhearing, and idle listening \\
\hline
\end{tabular}

\subsection{Classification of WSN MAC protocols}

By and large, WSN MAC protocols are classified into three general categories that are contentionbased, scheduling-based and hybrid MAC protocols as shown in Figure 1.

2.3.1Contention-based MAC protocols

Contention-based MAC protocols are designed based on carrier sense multiple access (CSMA). They are primarily designed with the capability detecting the state of the medium before transmitting through 214 listening otherwise known as carrier sense. These protocols have the advantage to exploit the full bandwidth of the communication channel [9]. Probabilistic coordination occurs in contention-based protocols as a result of the nodes' competition for a shared channel. These protocols have higher rates of collisions, overhearing and idle listening. SensorMAC (S-MAC) [7], timeout-MAC (T-MAC) [10], dynamic sensor-MAC (DS-MAC) [11], adaptive energy efficient-MAC (AEE-MAC) [12], 
synchronous receiver initiated-MAC (SRI-MAC) [13], Berkeley-MAC (B-MAC) [14], short preambleMAC (X-MAC) [15], convergence-MAC (C-MAC) [16], receiver initiated-MAC (RI-MAC) [17] and predictive wakeup MAC (PW-MAC) [18] are typical examples of this category. We classify them into two groups synchronous and asynchronous built on the nature of their algorithms.

2.3.1.1Synchronous MAC protocols

S-MAC [7] is designed to reduce idle listening, collisions, and overhearing by putting nodes in listen/sleep periods. Figure 2 shows that the listen periods in S-MAC is fixed while the duration of the sleep period depends on a predefined application based duty cycle factor. In S-MAC, the listen period is divided into SYNC and Data periods. During SYNC period, the node receives SYNC packet from its neighbours and store it. In the data period, exchange of data packets occurs which include request to send (RTS), clear to send (CTS), DATA, and acknowledgment (ACK) messages.

High latency occurs in S-MAC as a result of its fixed sleep periods; to solve this problem an adaptive listening mechanism was introduced. Therefore, when a node overhears an ongoing transmission from its neighbour, it will only wake up to receive a packet destined to it at the end of that transmission period else it will go back to sleep mode, some improvements are proposed in [2] to overcome the problems of S-MAC.

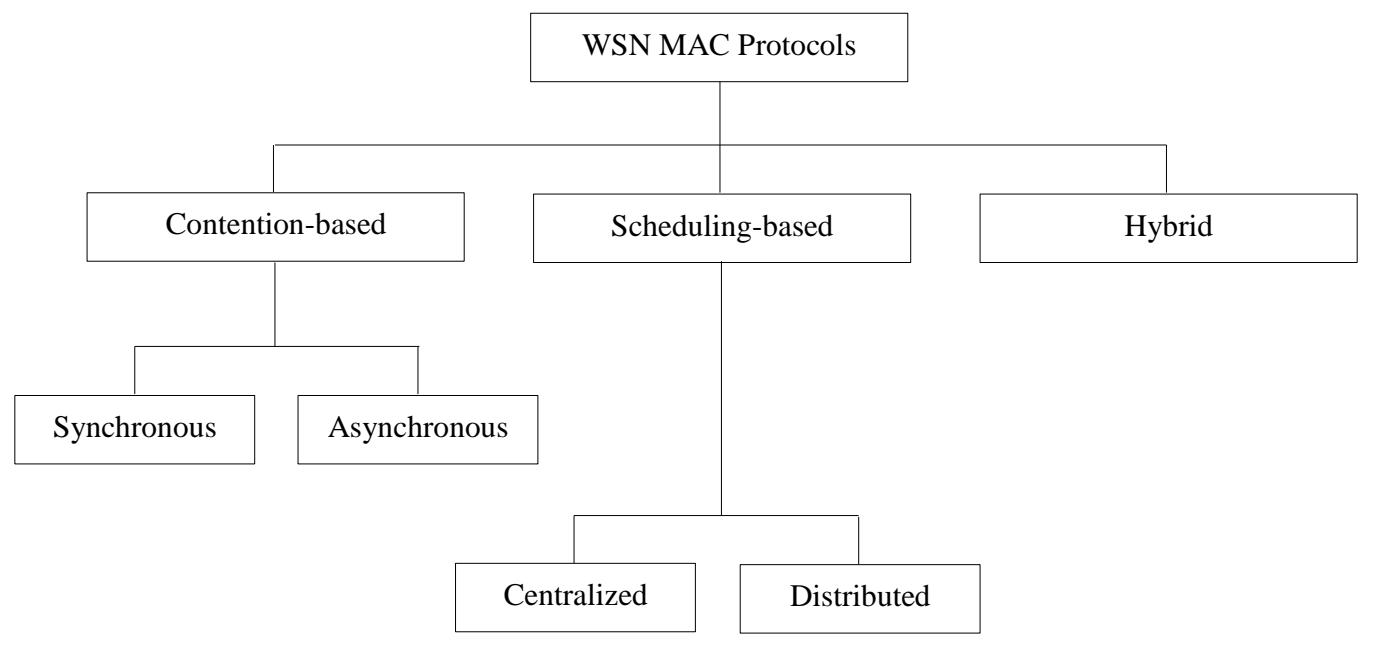

Figure 1 WSN MAC classification

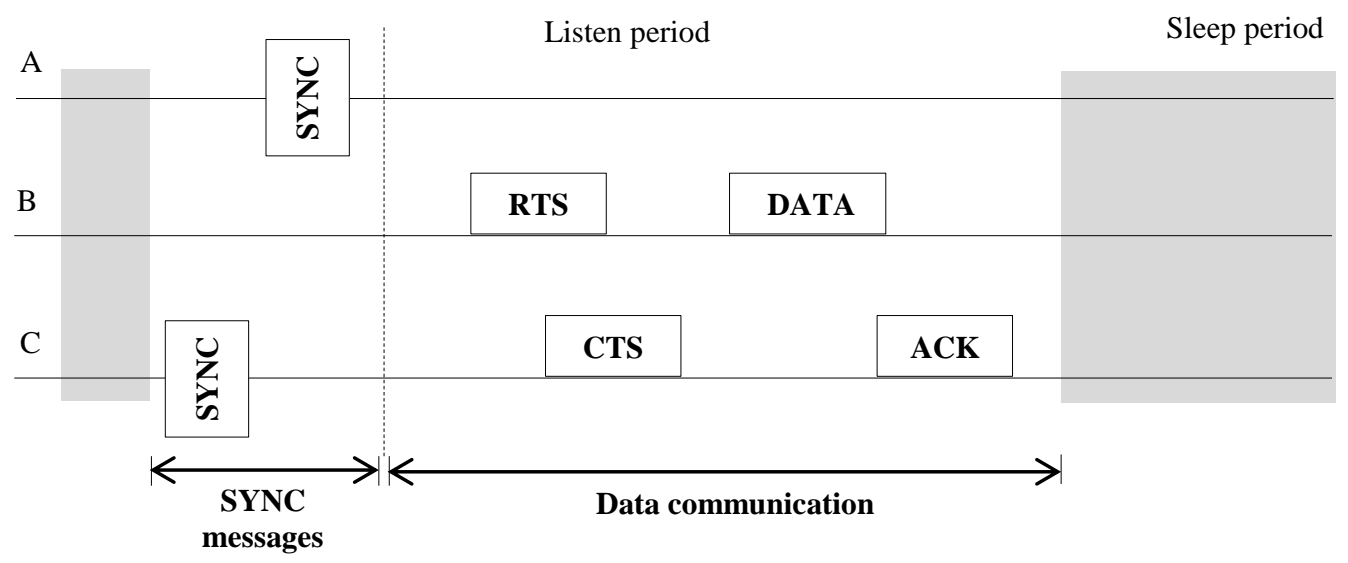

Figure 2 S-MAC periodic listen/sleep

T-MAC [9], was proposed to improve the energy efficiency of S-MAC especially under variable traffic condition and to solve the S-MAC's fixed duty cycle 215 by prematurely sending nodes back to sleep mode in the absence of any event for a given period known as 'Time Active (TA)' period. In T-MAC, nodes transmit messages in bursts. Similar to S-MAC, T- 
MAC also uses the RTS-CTS-ACK scheme. Better results are achieved with T-MAC under variable traffic. Both S-MAC and T-MAC uses SYNC message to schedule duty cycling and packet transmission; this requires a substantial amount of energy even in the absence of traffic.

T-MAC achieves better energy efficiency compared to S-MAC by reducing collisions and redundancy since nodes go back to sleep mode in the absence of any activity during the TA period at the detriment of high latency and reduced throughput. In T-MAC, packets are sent in bursts. As a result, the delay is minimized. However, T-MAC suffers from an early sleeping problem that was later solved by the introduction of future-request-to-send (FRTS).

DS-MAC [11], introduced dynamic duty cycle feature to S-MAC to reduce latency for delaysensitive WSN applications. Nodes share their onehop latency values within the SYNC period. In DSMAC, nodes begins transmission with the same duty cycle. At a point where a receiver node identified that a one-hop latency value is high, its sleep period is shortened and broadcasts it within its current SYNC period.

Consequently, when a sender node receives notice of a decrease in sleep-period, it performs a check for packets intended for that receiver node, if there is any packet destined for the receiver in its queue, the node than double its duty cycle whenever its battery level exceeds a fixed threshold. The duty cycle is doubled as such the schedules of the neighbour nodes will not be affected. DS-MAC achieves better latency compared to S-MAC [7] because it uses less frame duration it, therefore, achieves less throughput in high traffic. DS-MAC also achieves a better average power consumption per packet compared to S-MAC [7].

AEE-MAC [12] is proposed to optimize the energy efficiency. Similar to S-MAC [7], AEE-MAC uses duty cycling to save energy by avoiding idle listening. AEE-MAC decreases overhearing by putting nodes with no packets to sleep mode on receipt of CTS destined for other nodes. The protocol incorporates three optimization schemes, adaptive sleeping and reusing of the channel, the use of combined SYNC and RTS control packets and use of combined ACK and RTS control packets in a bidirectional and multi-hop data transmission. The adaptive sleeping and reusing of channel reduce the idle listening of S-MAC protocol by combining the durations of the communication in the control packets. The combination of SYNC and RTS control packets decides the actual network traffic load by setting nodes back into sleep mode if there are nodes with packets to transmit during the active period. While the combination of RTS and ACK control packets reduces overhead and collision while creating better channel utilization.

SRI-MAC [13], is a synchronous duty cycle protocol, it adopts the principle of receiver-initiated data transmission. In SRI-MAC, the receiver node transmits beacon signal advertising that it is awake and ready to receive data. The beacon comprises the receiver's id and the duration allocation period (DAP) which relies on the number of the receiver's neighbours. The value is used as a common factor to generate back-off values for collision avoidance. Each sender transmits an RTS packet comprising the node id, the id of the intended receiver and the data size upon receiving a beacon. The receiver will then transmit a CTS packet, which assigns time slots to senders that registered via the RTS packet. At this juncture, communication period starts and senders wake up based on the predetermined order. In an event where a receiver hears nothing from the sender, the receiver's beacon will go unanswered and thus no CTS will be transmitted. After passing an interval having a duration specified by DAP within the beacon, the channel will be considered idle by other potential receivers.

DAP mechanism induced an energy saving since only nodes taking part in a communication involve in the initial information period where the receiver announces itself and then can stay asleep for the remainder of the time slot.

2.3.1.2Asynchronous MAC protocols

B-MAC [14], uses asynchronous duty cycle and long preambles to wake up receivers. B-MAC uses an adaptive preamble scheme to achieve low power operation and minimal idle listening. A low power listening (LPL) scheme is used where a node cycle between awake and sleep periods. B-MAC uses the clear channel assessment (CCA) technique to check if there is a packet arriving when a node wakes up. While a node is awake, it listens for a long preamble to calculate if it needs to stay awake or switch back to sleep mode. This technique saves substantial amounts of energy. B-MAC significantly reduces idle listening and low overhead when the network is idle. B-MAC does not use overhead packets or any other control frame by default, but they can be added. The major 
setbacks in B-MAC are overhearing, long preamble as well as high latency and an increased contention.

X-MAC [15] is designed to improve on B-MAC [14] by further minimizing the issues of low power listening (LPL), overhearing and long preamble using strobe preamble that allows interruption and fast wake up of nodes. A strobed preamble is used to allow a receiver to interfere with a long preamble on discovering that it is the target receiver on wake up. Strobed preamble helps in reducing the consumption of time and energy while awaiting the whole preamble to complete. X-MAC achieves significant energy efficiency and low latency as a result of the short preamble as well as a low overhead. This protocol also has the disadvantage that preamble transmission still inhabits the channel up to the time when the target receiver wakes up.

C-MAC [16] is a protocol proposed to improve WSN energy efficiency and the latency. The protocol is equipped with three modules, the Aggressive RTS, anycast and convergent packet forwarding mechanisms. The aggressive RTS is furnished with double channel check for channel assessment. The protocol circumvents synchronization overhead while supporting low latency. In the absence of traffic, the protocol operates at very low duty cycles. In the state of traffic, C-MAC uses anycast-based packet forwarding to wake up forwarding nodes or speedily determine a forwarder and then converges from route-suboptimal anycast with asynchronous duty cycling to route-optimal unicast with the synchronized scheduling scheme. For flow initialization, C-MAC uses anycast and uses convergent packet forwarding for flow stabilization. After convergence, nodes can use the synchronized wake up schedule to preserve more energy. C-MAC achieved a high throughput, low latency and consumed less energy. However, it is only designed for low duty cycle applications.

RI-MAC) [17] is an asynchronous MAC protocol that sets communication depending on receivers. Nodes periodically wake up based on their schedule, if the medium is idle after a receiver node wakes up, it broadcasts a beacon message to potential senders. After receiving the beacon, the sender immediately transmits a data packet to the receiver. RI-MAC achieves an excellent latency performance by minimizing the rendezvous time occupied by the sender and receiver in a wireless channel. Additionally, the contention is effectively resolved at the receivers; therefore RI-MAC can effectively reduce overhead. RI-MAC is efficient in detecting collisions and data frames recovery more than BMAC [14] and X-MAC [15] where the senders are hidden to each other.

Moreover, RI-MAC can use beacon with two roles ACK and ready to receive. Consequently, RI-MAC outpaces the earlier stated protocols in all aspects. RIMAC, however, poses some disadvantage at senders. In a case where a sender with awaiting packet needs to keep its radio on until a potential receiver wakes up. Few protocols were proposed to address this problem.

PW-MAC [18] is another asynchronous MAC protocol where senders guess the receivers' wake-up time, queue packets and wake up right before the guessed time to minimize node energy consumption. PW-MAC employs the receiver-initiated method because of its good receiver duty cycle performance. The protocol offers a communication mechanism which can be used for transmitting the prediction state of a node. The protocol uses an on-demand prediction error correction mechanism which helps to correct timing challenges caused by volatile hardware, operating system delays, and clock drift. PW-MAC also presents an effective prediction based re-transmission mechanism to attain excellent energy efficiency even if collisions occur, and packets must be re-transmitted.

The predictable wake-up times are used to increase performance in the event of collisions as well as channel errors. When there is a need for retransmission, sender nodes in RI-MAC [17] stay awake until receivers wake up again, unlike in PWMAC, senders only wake up at the next predicted receiver's wake up time; this significantly minimizes the energy consumption while waiting for a receiver to wake up. Some of the shortcomings of PW-MAC are overhead caused by the beacon and idle listening compared to other protocols such as X-MAC [15] and RI-MAC [17].

2.3.2 Schedule-based MAC protocols

These protocols are designed according to the principle of time division multiple access (TDMA) scheduling to carry out communication. In this type of approach, the schedule is established to allow nodes access a channel and communicate with each other. Synchronization is required between nodes to ensure a shorter idle listening. Nodes share the same frequency by dividing the time into frames; each frame is then divided into slots [19-21]. Nodes transmit in rapid sequence using assigned time slot. 
Collision reduction and fairness among different nodes and the increase in overall throughput are the main goals of this approach. Some schedule-based MAC protocols such as low-energy adaptive clustering hierarchy (LEACH) [22], (priority-based MAC protocol for wireless sensor networks) PRIMA [23], data gathering-MAC (D-MAC) [24], power efficient and delay aware MAC protocol (PEDAMACS) [25], dynamic energy efficient TDMA-based-MAC (DEE-MAC) [26], traffic adaptive MAC (TRAMA) [27], node activation multiple access (NAMA) [28], power aware clustered TDMA (PACT) [29], self-organizing-MAC for sensor networks (S-MACS) [30], lightweight MAC (LMAC) [31], and advanced-MAC (A-MAC) [32] are examined and presented.

We classify the scheduling-based protocols into two categories based on the nature of their algorithms: centralized and distributed protocols. The centralized scheduling-based protocols use cluster heads $(\mathrm{CH})$ or base station (BS) to manage their schedules centrally and assign a time slot to all nodes within the network. While in the distributed scheduling protocols, nodes manage their respective schedules based on the local information they obtained. Therefore, there is no need for any centralized entity such as $\mathrm{CH}$ or BS.

2.3.2.1Centralized scheduled based protocols The LEACH [22] is a schedule based protocol that splits similar WSN into clusters. Each cluster is managed by a cluster head, which is in charge of creating and maintaining schedules, interacting with cluster members, and forwarding of messages to the sink node. Since cluster head needs to always stay on, the cluster head to run out of battery earlier is highly likely. Although, LEACH adopts a randomized rotation mechanism for choosing a cluster head. Each node can become the cluster head, but preference is given to the node that has not been a cluster head for a prolonged period. LEACH works in rounds, each further divided into the set-up and steady-state phases. The cluster formation takes place during the set-up phase; each cluster head transmits an advertisement (ADV) packet using CSMA to invite its cluster members. The cluster head, then generates and broadcasts a TDMA schedule for nodes that have sent join-request (REQ) to it. To reduce inter-cluster interference, the cluster head selects a random codedivision multiple access (CDMA) code node within its cluster.

When the setup phase is over, the steady-state phase starts, and a node can transmit data to its cluster head using the allocated slot. When the cluster head receives packets from its members, it aggregates and sends them to the sink node. LEACH guaranteed that each member node belongs to at most one cluster. However, due to an ADV collision, it cannot guarantee that each member node belongs to the cluster. The protocol considers that all nodes fall within the communication coverage of the sink. The absence of such multi-hop communication abilities within LEACH hinders the scalability of the network. Moreover, LEACH comes with the assumption that nodes always have data ready for transmission during their allotted time, hence the occurrence of channel under-utilization. Perfect correlation among nodes is assumed, which is hardly possible in WSNs.

PRIMA [23] uses a similar approach as that of LEACH [22] in forming clusters of nodes as well as selecting cluster heads for managing communication and synchronization within each cluster. In PRIMA, cluster heads interchange every 15 minutes. The protocol assigns four different priorities for information, via instructing the application layer to append two bits at the end of every packet. The MAC layer consists of two different protocols, classifierMAC (C-MAC) and channel access-MAC (CA$\mathrm{MAC}$ ). The $\mathrm{C}-\mathrm{MAC}$ is responsible for assigning packets to the four priority queues by their respective priorities. While the CA-MAC grants the channel access using carrier sense multiple access with collision avoidance (CSMA/CA) and TDMA slots. The CSMA phase creates schedules while the TDMA phase is used for collision-free packet transmission.

PRIMA reduced packet delivery delay based on traffic requirements. The protocol also increases the network lifetime through cluster head rotation. However, similar to LEACH [22] when a cluster head dies, all nodes in that cluster are inoperable until the new cluster head is selected. Also, overhead packets in the CSMA phase increase the energy consumption.

D-MAC [24] is designed for tree-based multi-hop topology for energy efficiency and latency reduction. As shown in Figure 3, based on naturally formed data gathering tree, packets can flow continuously to the sink because of the staggered active/sleep schedules of the nodes. The node's schedule is divided into three phases, receiving, sending and sleeping. The receiving node receives the packet and sends ACK, the sending node forwards packet to the next hop and receives ACK while the sleeping node turns off its radio. A variable $\mu$ represents the length of time required for transmission and reception of 
one packet. Nodes of equal depth have the equal offset, as such, they contend for transmitting to their respective receivers. In a situation where one node wins the channel access, neighbouring nodes of the same level lose their chance of transmission.

A scheme called data prediction and more-to-send notification are introduced to increase the number of active slots. In D-MAC, the RTS/CTS control packets is not used to avoid needless overhead considering the relativity of small packet size in WSN applications. The D-MAC protocol achieves a good latency in comparison to other sleep-listen dutycycle methods.

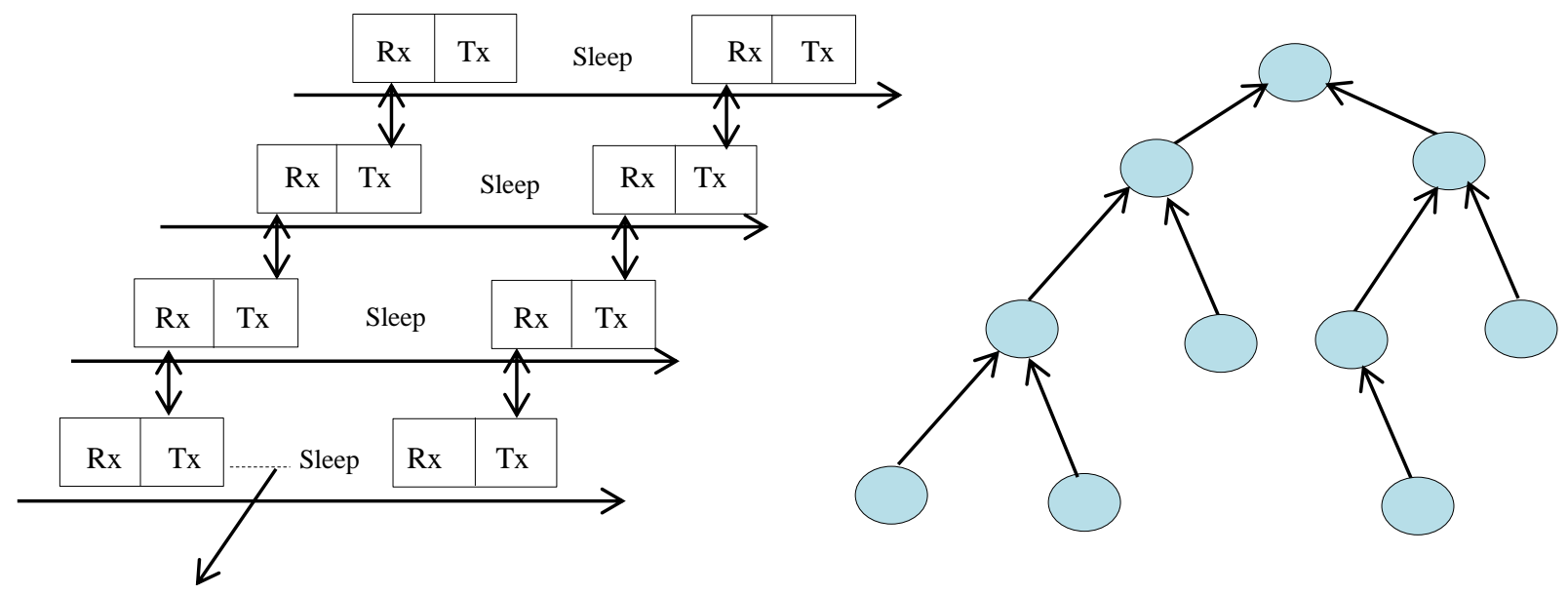

More active slots if required

Figure 3 D-MAC data gathering tree scheme

Nevertheless, this approach faces challenges of packet collision that occurred as a result of shared schedule among the nodes. Therefore, D-MAC performs better in a network where the transmission paths and transmission rates are known and do not experience sudden changes.

PEDAMACS [25] is another schedule-based protocol for WSNs. It extends the common single-hop TDMA to the multi-hop sensor network. It uses a strong access point (AP) called 'sink' to synchronize the nodes as well as schedule their transmissions and receptions. They defined three transmission power levels to reach three distances: Pl, maximum, Pm, medium and Ps, minimum. The protocol has four phases namely, the topology learning, topology collection, scheduling phase and the adjustment.

The AP gathers the topology information, and the scheduling algorithm determines the transmission and receiving periods for each node. All Nodes send their topology information to the AP using Ps to enable data to go through many hops. Then the AP announces the transmission schedule to other nodes, and every node adjusts its clock to tally with the time slots allocated for transmitting and receive. At the end of the scheduling phase, nodes transmit adjustment topology packets to indicate any changes in neighbours or interferers. PEDAMACS has the advantages of energy saving, increase throughput, and guaranteed end-to-end delay. It major setbacks is reliability. Thus, the protocol is not suitable for realtime applications that need guaranteed reliability. However, PEDAMACS has additional overhead packets apart from the RTS/CTS and ACK packets.

DEE-MAC [26] proposes to reduce energy consumption by compelling the idle listening nodes to sleep. Clusters are formed based on the remaining power as all nodes compete to become the cluster head. DEE-MAC operations consist of rounds; each round contains two phases, cluster formation, and transmission. During the cluster formation phase, a node becomes the cluster head based on its remaining power level; a node with highest power level becomes the cluster head. The transmission phase comprises of numerous sessions; each session has a contention and transmission period. In the contention period, nodes keep their radio on and indicate interest to transmit the packet to the cluster head. The cluster head builds and transmits a TDMA schedule to all 
nodes assigning them one slot in each session for either receiving or transmission data.

These techniques of clustering and TDMA scheme significantly reduces idle listening in large-scale WSNs. Further improvement in energy efficiency may be achieved by investigating the likelihood of error in a packet within a contention period, and by using inter-cluster communication through the nodes instead of cluster heads.

2.3.2.2Distributed scheduled based MAC protocols

TRAMA [27] is a TDMA-based protocol that uses a traffic adaptive distributed election scheme which selects receivers by announced schedules by transmitters. TRAMA consists of three components, neighbor protocol (NP), schedule exchange protocol (SEP) and adaptive election algorithms. The neighbour protocol (NP) transmits one-hop neighbour information among the neighbouring nodes to obtain consistent two-hop topology information across all nodes using the signalling slots. Exchange of trafficbased information or schedules between neighbouring nodes is carried out through the SEP. To achieve collision-free transmission, selection of transmitters and receivers is performed by the adaptive election algorithm (AEA) using the information obtained from NP and SEP.

NAMA [28] where each time slot uses a distributed election algorithm to select one transmitter in each two-hop neighbourhood by adopting the neighbourhood-aware contention resolution (NCR). TRAMA switches between random access period and scheduled access period. Nodes that have data to send will claim slots for use in random access.

TRAMA achieves significant energy efficiency and throughput by ensuring a collision-free transmission and its ability to switch nodes to the idle state when they are not active. Its major challenge is long delay compared to other protocols as well as high latency in a high traffic network.

PACT [29] is a TDMA MAC protocol that used passive clustering to prolong battery and network lifetime. The principle of passive clustering takes into consideration the individual energy level of every node and appoints so-called "cluster heads" depending on how long a battery lifetime is left. The task of being clustered head is allotted to the nodes with the biggest energy reserves, but is rotated as soon as this ratio change, to preserve a minimum of energy in all sensor nodes. The subset of cluster heads and certain gateway nodes that are accountable for traffic between neighbouring clusters have priority in allocating time slots. Only after they have received their demanded bandwidth, the ordinary sensor nodes are granted the possibility to choose between the remaining time slots. Energy consumption is further minimized by adjusting the active periods of the nodes to the data traffic present in the network at a certain time, i.e. nodes are shut down when there is no transmission concerning them. PACT can prolong the network lifetime up to five times compared to the IEEE 802.11 wireless standard due to a more efficient power management. Through the use of passive clustering, network lifetime can also be improved by increasing the sensor node density in the network.

S-MACS [30] protocol uses a combination of TDMA and frequency division multiple access (FDMA) or CDMA for medium access. Nodes in S-MACS requires no global synchronization or clustering to establish reception/transmission schedules with their neighbours. S-MACS combines the neighbour discovery phase with the channel assignment phase. It assigns the channel with a link immediately after the link is discovered, instead of waiting to finish the network-wide neighbour discovery process. Nodes in S-MACS operate on a random FDMA or CDMA code to reduce collisions between neighbouring links. Each node regularly performs neighbour discovery procedure and establishes a directional link with the discovered neighbours by assigning a time slot to the link. Nodes in S-MACS use TDMA-like superframe for communication with known neighbours.

S-MACS employs a local scheme instead of a global assignment to avoid computation and overhead in transmitting neighbour information to the sink node. In S-MACS, the time slots is lost if the sensor node has no data to send to an intended receiver.

LMAC [31] protocol minimizes the number of transceiver switches, to make the sleep interval of sensor nodes adaptive to the amount of data traffic. A transmitted packet in LMAC consists of two parts: the control and data part. The control packets are of fixed size and contained the ID of the time slot owner it also indicates the distance from the transmitting node to the gateway for easy routing. The control packet data are also used in maintaining synchronization between nodes.

All neighbouring nodes focus more on receiving control packets from their neighbours. When a packet is not addressed to a node or the packet is not 
addressed as an omnicast, the nodes will switch off their transceivers to save energy, only to wake at the next time slot. Otherwise, if a node is addressed, it will listen to the data unit that might not use the entire remainder of the time slot. After successful packet transfer, both transmitter and receiver(s) turn off their transceivers.

LMAC achieves a significant energy saving and prolongs network lifetime. Nodes in the network can communicate with each other in a collision-free manner. However, idle listening increases LMAC since nodes always listen to the control part of all slots in a frame, to allow nodes receive data and to allow new nodes to join the network anytime. As the slots are computed only once, this protocol is not suitable for mobile sensor networks, where nodes can enter and leave the other nodes' radio neighbourhood at any time.

A-MAC [32] adapted from LMAC [31] A-MAC is developed for a low rate and reliable data transportation to prolong the network life of WSN. The protocol uses a distributed technique for time slot allocation by gathering its neighbourhood information, unlike other conventional TDMA-based protocols that depend on a central controller for time slot allocation. A-MAC manages energy using scheduled power down mode in the absence of any event.

The protocol is designed in several frames; each frame consists of several time slots. Nodes transmit a beacon at the commencement of their time slots, for synchronization and neighbour information exchanges. The controlled node tells which node will be participating in next data session to stay in listening mode while other nodes go into power down mode until the end of the current time slot.

Slots assignment in A-MAC is divided into four states; initial, wait, discover and active. A node in the initial state, listen to neighbours beacon for synchronization with the network, and then randomly choose a number of waiting for frames and enter the into the wait state. A node entered the discovery state when the waiting counter expired and started collecting its neighbourhood information by listening to the beacon messages from its neighbouring node for listen-frame frames. A node enters an active state when it successfully selects a time slot. Nodes enter sleep mode in two situations, first, after transmitting a beacon message and no more data packet scheduled to be transmitted and second, if received a beacon message from its neighbouring node indicates no incoming data packet. Compared to LMAC [31], AMAC allows a node to transmit to multiple destinations.

\subsubsection{Hybrid MAC protocols}

The hybrid MAC protocols combine the strengths of contention-based and scheduling-based protocols while minimizing their weaknesses, to produce a dual characteristic protocol and achieve better performance. Examples of hybrid MAC protocols are Zebra-MAC (Z-MAC) [33], Funneling-MAC [34], A-MAC [35], Crankshaft MAC [36] and IEEE 802.15.4 [37].

Z-MAC [33] is a hybrid WSN MAC protocol that combines the strengths of TDMA and CSMA while compensating for their weaknesses. Z-MAC is regarded as traffic adaptive protocol because of its ability to switch to CSMA under low contention to achieve excellent channel utilization and low delay. It switches to TDMA under high contention to avoid a collision and to achieve high channel utilization and fairness. Nodes using Z-MAC can utilize slot assigned to another, unlike in the traditional TDMA scheme. Priority is always given to the owner of the slot over the non-owners, to avoid possible collisions.

Z-MAC uses distributed randomized time slot scheduling algorithm (DRAND) [38] to assign slots to nodes. DRAND is run during setup phase and guarantees that no two nodes within a two-hop neighbouring are allocated the same time slot. During the transmission mode, time is divided into slots. ZMAC uses CSMA as the baseline MAC scheme and a TDMA schedule to enhance contention resolution. The protocol also offers a simple two-hop synchronization technique which allows the sender to vary its frequency according to the current data rate and resources. Therefore, Z-MAC is known to be robust enough to withstand timing and slot assignment failures, channel condition issues, and dynamic topological infrastructure.

There is one drawback with Z-MAC in that it requires global time-synchronization during the startup phase. This unarguably consumes energy, time, and memory for lightweight nodes. A highly dynamic WSN may need to perform this costly procedure more than once. Moreover, the complexity of maintaining both CSMA and TDMA modes, as well as switching between the high contention and low contention states, contention and possible collisions among nodes in gaining control to the slots owned by 
others, and bandwidth under-utilization contribute to some other issues of Z-MAC.

Funneling-MAC [34] is a hybrid, localized and sinkoriented protocol that is mainly a CSMA/CA with a TDMA scheduling managed by the sink node and operates locally in the funneling region. It reduces the funneling effect in WSN by reducing traffic in the funneling region to increase the network lifetime. All nodes in funneling-MAC operate in the CSMA mode by default. A beacon broadcast activates the TDMA scheduling from the sink node. Nodes that receive the beacon consider themselves within the funneling region and are called Fnodes. These nodes can perform TDMA and switch back to CSMA if they do not receive the beacon within a time interval. The sink node calculates the TDMA schedule as per traffic conditions. Each Fnode transmits its scheduled packet during the allocated time slot specified in the TDMA frame.

The sink allocates slots to each path using information in the path table. Schedule packet contains path head id of scheduled paths, the number of slots assigned to each path. Fnodes receive schedule packet and check which slots are assigned to them. Fnodes keep path table containing path head id of each path going through it, the number of hops to path head. Using this fnode compute which slot assigned to itself. F-nodes transmit packet in their slot in TDMA frame. Even though it assigns time slots only to nodes that are on active routes, it cannot manage the nodes that are out of the sink communication range.

In funneling-MAC, fnodes waste more energy in receiving beacons and schedule frames due to the high power demand for sending the beacon and the schedule frames. The protocol also bears complexity in managing the funneling region over the time and in performing CSMA after every second TDMA frame. The number of Fnodes in a dense WSN could be very high, as a result, each Fnode has to wait longer for its turn to communicate with the sink, hence the increase in network latency.

A new MAC A-MAC [35] is a hybrid protocol based on CSMA and TDMA schemes proposed to provide collision-free, non-overhearing and less idle listening transmission services in WSN. A-MAC uses TDMA as its baseline MAC with CSMA for accessibility of the wireless channel. The protocol is designed for long-term surveillance and monitoring systems. AMAC allocates unique time slots to each node within its two-hop neighbours. Nodes use the pre-assigned time slot for data transmission without interference. The protocol also has an advertisement scheme used by sending nodes to notify their neighbours about their transmission schedule. Using this scheme, only the receiver node needs to be active during the transmission period while other nodes to go to sleep.

Nodes using this protocol stay inactive most of the time until an event it detected. Even though an additional latency may be introduced, yet the network lifetime is dramatically prolonged. In A-MAC, nodes are notified of becoming receivers in advance. A node becomes active only when it is a sender or a receiver, during other periods the node goes to sleep. This technique drastically saves energy wastage caused by overhearing and idle listening.

Crankshaft MAC [36] is another hybrid MAC protocol, designed specifically for highly dense wireless sensor networks. Crankshaft employs node synchronization offset wake up schedules to combat overhearing by neighbouring nodes. The protocol divides time into frames; each frame is split into two slots: the broadcast and unicast slots. During the broadcast slot, all the nodes wake up to listen to an incoming message. If required to transmit a message, a node contends with other nodes in the contention window to gain control of the slot. Once successfully accessed the medium, it uses the destination node's MAC address to determine the wake-up slot of the destination node before start transmitting.

Energy saving is achieved by using efficient channel polling and contention resolution techniques. The protocol uses a channel polling mechanism identical to the scheduled channel polling-MAC (SCP-MAC) [39] in which only senders are awake during the contention period, and only winners of the contention will be awake for the receiver's poll. The receiver polls the preamble sent by the sender at fixed offsets from the beginning of the frame and gets a rendezvous of the message transmission. Both sender and receiver depend on the rendezvous for transmitting and receiving messages in the message exchange window. Crankshaft utilizes a Data/Ack sequence for unicast messages.

In dense networks, Crankshaft protocol can offer an excellent converge cast performance at minimal energy consumption, therefore becoming apt for monitoring applications in which energy efficiency is important. Crankshaft protocol has an inflexible 
structure, which restricts the applicability of the protocol in many applications.

Low-rate wireless personal area networks (LRWPAN) (IEEE 802.15.4 MAC) [37] the Institute of Electrical and Electronics Engineers (IEEE) Task Group 4 (TG4) together with the ZigBee Alliance proposed and developed a protocol stack for a LRWPAN. IEEE 802.15.4 equipped with a duty cycle mechanism where the size of active and inactive parts can be adjusted during the PAN formation. Although the protocol was not entirely designed for WSNs, it features such as simplicity, low rate, low power, and low-cost communication made it a strong contender for the WSN communication stack. It combines the contention based channel accessing scheme with the scheduling based on.

The beacon mode of IEEE 802.15.4 MAC defines a superframe structure to organize the channel access and data exchanges. The active period is divided into 16-time slots. A beacon frame is transmitted in the first timeslot followed by two other parts, the Contention Access Period (CAP) and the ContentionFree Period (CFP) which occupy the remaining time slots. The CFP part is also known as Guaranteed Time Slots (GTS) and can utilize up to 7-time slots. The span of the active and inactive periods, as well as that of a single time slot, is configurable and traffic dependant. Data transmissions occur either in CAP or GTS periods. Data communication is achieved in CAP using slotted CSMA-CA while in the GTS nodes are allocated fixed time slots.

To achieve energy efficiency in IEEE 802.15.4 MAC, nodes are put into sleep mode during the inactive period and when there is no data to transmit or received from the coordinator. However, the coordinator took the weight of energy cost, because the coordinator has to be active during the entire active period.

\subsubsection{QoS-based MAC protocols for WSNs}

QoS provisioning is spread across the 7 OSI layers depending on the layer capability. Each layer has specific parameters used for performance evaluation and QoS assessment [40].

Our greatest concern is the MAC layer, which the performance of all upper layers depends upon. QoS support in other layers cannot be achieved without a MAC protocol that can resolve the issues of medium sharing and supports reliable communication [41]. This makes QoS provisioning in the MAC layer of significant importance for overall QoS in WSN.
QoS-aware-MAC (Q-MAC) [42] is an energyefficient and priority-based QoS-aware media access control protocol. Q-MAC tries to curtail energy consumption at the same time providing QoS. QMAC adopts the multiple access with collision avoidance for wireless (MACAW) protocol as an underlying protocol to access the wireless channel. Q-MAC introduces a new queuing model that sets priority levels for different queues to reveal the criticality of data packets coming from different sensor nodes in order to fulfil QoS requirements for diverse traffic types. Higher priority queues are provided preference over low priority queues. QMAC comprises of intra-node and inter-node scheduling in WSN. The intra-node scheduling scheme implements a multi-queue first-in first-out (FIFO) based approach to classifying data packets depending on their application and MAC layer abstraction while the inter-node scheduling manages channel access aimed at minimizing energy consumption through reducing collision and idle listening. The major drawback of this protocol is higher latencies.

Saxena et al. [43], proposes a QoS for multimedia transmission in WSN. The protocol uses CSMA/CA approach with three types of traffic classes, streaming video, non-real-time and best effort. The protocol adaptively updates the contention window size as well as duty cycle, according to different coefficients of each traffic class and the dominant traffic class respectively. The protocol achieved better energy conservation. However, it introduces a significant overhead. Also, idle listening and early sleeping problems may likely occur due to the absence of local or global synchronization between sensor nodes. Also, lower priority packets suffer from high latency.

Differentiated services-MAC (Diff-MAC) [6] is proposed as a different approach of intra-node packet prioritization and contention window $(\mathrm{CW})$ adaptation. In Saxena et al. MAC [43] one FIFO queue per traffic class is implemented while DiffMAC provides fair prioritization of packets within the same class based on hop count metric of each packet and uses a weighted fair queuing (WFQ) to control the relative throughput of each traffic class. The two protocols use similar mechanisms to IEEE 802.11e standard, especially on medium access prioritization. Diff-MAC achieves fairness among the different traffic classes. Not efficient under high contention due to RTS/CTS exchanges that consume extra bandwidth. 
Priority-based QoS MAC (PQMAC) [44], utilizes the features of contention-based and schedule-based approaches as well as a hybrid scheme for medium sharing. Slot assignment, global clock synchronization, and neighbour discovery are done during the setup phase followed by the transmission phase.

The slot assignment considers the two-hop distance neighbour nodes and allots different time slots using DRAND [38] algorithm, the time frame rule determines the frame size as in Z-MAC [33] protocol. Slot owner has an exclusive right to send the data within its allocated slot time. If the slot owner does not have data to transmit, or has a lower priority data, non-owners can contend for the slot and transmit based on the priorities of their data.

In PQMAC, data is divided into classes based on its scenario, application, and transmission type. Every class has its priority level. High-priority data are placed into a high-priority queue and low-priority data are placed into a low-priority queue. Highpriority data always have the opportunity to be sent during any listen time. PQMAC uses three schemes for QoS provisioning, doubling scheme, advance wake up and dynamic priority listen-scheduling for additional listen time for high priority data, calculating node's probability of receiving highpriority data and changing the listen/sleep state of nodes respectively. The simulation indicated that PQMAC reduces latency and conserves energy as well as improved channel utilization and significantly reduces the probability of collision.

\section{Discussion}

Contention-based MAC protocols as the name implies, contend for medium access whenever a sensor node wants to communicate with peers. Contention-based MAC protocols are robust and scalable; they easily adapt to traffic conditions. Unfortunately, their energy expenditure is high due to contention, collisions idle listening, overhearing, and over-emitting.

On the other hand, scheduling-based MAC protocols are designed based on TDMA, FDMA, and CDMA schemes; the protocols pose significant advantage of energy efficiency since nodes only turn on their radio during their allocated time slot and sleep for the rest of the time, this removed contention and reduced the probability of possible collision. However, the protocols have the disadvantages which offset their energy efficiency. Protocols in this category have 224 limited scalability and adaptability. In an event where there is a new node joining the network, both the frame size and time slot assignment are required for reconfiguration of the network to adapt to the change. Also, the maintenance of frame synchronization creates additional latency and reduced throughput which resulted in unnecessary energy consumption.

The hybrid MAC protocols complement the contention and scheduling schemes at the same time offsetting their disadvantages. Contention schemes, offers robustness, flexibility, simplicity, and low latency, therefore allowing nodes to adapt to changes in the network topology supported by some control signals. In the contrary, scheduling schemes possess features like high channel utilization, collision-free, and throughput, appropriate for high traffic load conditions. The protocols in this category use a mechanism that switches between the contention and scheduling schemes whenever the need arises.

Even though hybrid MAC protocols appear to be an idyllic method to accomplish optimum performance of the MAC protocol requirement, this benefit comes at the detriment of protocol complexity, in this case a precise mapping from traffic loads to the switching point might not be achieved simply.

The QoS provisioning goal for all layers of the communication protocol stack is equal, since each layer strived to provide the anticipated QoS level to applications and users while reducing energy consumption, and accordingly prolonging the network lifetime. The efficiency of the QoS provisioning mechanism at individual layers relies solely on the layer capabilities. QoS metrics usually include: bandwidth, throughput, latency, jitter, packet loss and network availability. However, current energy consumption is becoming a standard part of QoS metrics considering its relevance in the overall WSN design.

In reality, there are various other factors to be considered when designing MAC protocols. Other than energy efficient and QoS-aware, sensors topology [45, 46], mobility patterns [47], energy harvesting [48] and security aware [49, 50] are examples of issues that could affect the performance and lifetime of a sensor and sensor network.

\section{Conclusion}

We conduct a brief survey of WSN MAC protocols. First, we introduce the concept of WSNs and its application. Secondly, we described the MAC, causes 
of energy waste in WSN and the attributes of good MAC design. We also presented the classification of MAC protocols for WSN with detailed discussion on contention-based, scheduling-based and hybrid MAC protocols. In contention-based protocols, we discussed in detail the two general sub-categories of synchronous and asynchronous. We investigated each sub-category and the protocols that belong to it, highlighting its advantages and disadvantages. Also, in the scheduling-based protocols were discussed in detail, we also classified them into two subcategories, the centralized and distributed algorithms. Lastly, we presented the hybrid protocols where we examine their cons and pros.

After discussing several protocols from each category, we have presented a meaningful comparison of these protocols on different performance metrics, the comparison in Table 2 shows that the majority of the protocols leaned towards energy conservation. Other parameters are either supported partially or trade-off for another parameter. Latency (L), throughput $(\mathrm{T})$, bandwidth utilization, channel utilization are not considered in the design of most of the protocols. It is imperative that energy domain has gotten a vital breakthrough with the advent of other modes of energy saving such as energy harvesting techniques.

Since energy efficiency is fundamental for WSNs lifetime, it should have a greater concern than other parameters. However, in real-time, multimedia and other critical WSNs, more concern should focus on bandwidth, reliability and response time. Meanwhile, QoS parameters such as bandwidth, delay, jitter and packet loss are inversely proportional to the energy consumption, there is a need to trade-off between energy consumption and QoS provisioning.

Currently, QoS provisioning is application-specific, mostly in real-time, multimedia and critical WSNs that require a guaranteed performance to function appropriately. However, due to an increasing number of users and applications, QoS will no longer be optional in WSNs, rather it must become a standard part of WSNs. We, therefore, believe that QoS metrics such as bandwidth, delay, jitter and packet loss may play a critical role in the design of future MAC protocols for WSNs to ascertain QoS support.

Table 2 Comparison of different MAC protocols on various performance metrics

\begin{tabular}{lllll}
\hline Protocol & Access & Energy efficient & L & T \\
\hline S-MAC [7] & CSMA & Medium & Medium & Low \\
T-MAC [10] & CSMA & High & High & Low \\
DS-MAC [11] & CSMA & Medium & Low & Low \\
AEE-MAC [12] & CSMA & Medium & - & - \\
SRI-MAC [13] & CSMA & High & - & Low \\
B-MAC [14] & CSMA & Medium & High & Low \\
X-MAC [15] & CSMA & Medium & Low & High \\
C-MAC [16] & CSMA & Medium & Low & Low \\
RI-MAC [17] & CSMA & Medium & High & - \\
PW-MAC [18] & CSMA & High & - & - \\
LEACH [22] & TDMA & Medium & - & High \\
PRIMA [23] & TDMA & High & Low & High \\
D-MAC [24] & TDMA & Low & Low & High \\
PEDAMACS [25] & TDMA & Medium & Low & High \\
DEE-MAC [26] & TDMA & Medium & - & - \\
TRAMA [27] & TDMA & Low & Low & - \\
PACT [29] & TDMA & High & Low & - \\
S-MACS [30] & TDMA & Medium & - & - \\
LMAC [31] & TDMA & Medium & - & High \\
A-MAC [32] & TDMA & Medium & - & High \\
Z-MAC [33] & CSMA/ TDMA & Medium & - & - \\
Funneling-MAC [34] & TDMA/ CSMA & High & - & High \\
A-MAC [35] & CSMA/ TDMA & Medium & - & - \\
Crankshaft MAC [36] & CSMA/ TDMA & High & - & Low \\
IEEE 802.15.4 [37] & CSMA/ TDMA & Medium & & \\
\hline
\end{tabular}




\section{Acknowledgment}

The authors are grateful to the anonymous referees who have contributed to improving the quality of this paper. This research was supported by the Malaysian Ministry of Higher Education [Grant No: FRGS/1/2017/ICT03/UNISZA/02/1].

\section{Conflicts of interest}

The authors have no conflicts of interest to declare.

\section{References}

[1] Balen J, Zagar D, Martinovic G. Quality of service in wireless sensor networks: a survey and related patents. Recent Patents on Computer Science. 2011; 4(3):188202.

[2] Naik P, Sivalingam KM. A survey of MAC protocols for sensor networks. In wireless sensor networks 2004 (pp. 93-107). Springer, Boston, MA.

[3] Suriyachai P, Roedig U, Scott A. A survey of MAC protocols for mission-critical applications in wireless sensor networks. IEEE Communications Surveys \& Tutorials. 2012; 14(2):240-64.

[4] Demirkol I, Ersoy C, Alagoz F. MAC protocols for wireless sensor networks: a survey. IEEE Communications Magazine. 2006; 44(4):115-21.

[5] Matin MA, Islam MM. Overview of wireless sensor network. In wireless sensor networks-technology and protocols 2012. InTech.

[6] Yigitel MA, Incel OD, Ersoy C. QoS-aware MAC protocols for wireless sensor networks: a survey. Computer Networks. 2011; 55(8):1982-2004.

[7] Ye W, Heidemann J, Estrin D. An energy-efficient MAC protocol for wireless sensor networks. In proceedings of computer and communications societies 2002 (pp. 1567-76). IEEE.

[8] Ye W, Heidemann J, Estrin D. Medium access control with coordinated adaptive sleeping for wireless sensor networks. IEEE/ACM Transactions on Networking. 2004; 12(3):493-506.

[9] Casari P, Zorzi M. Protocol design issues in underwater acoustic networks. Computer Communications. 2011; 34(17):2013-25.

[10] Van Dam T, Langendoen K. An adaptive energyefficient MAC protocol for wireless sensor networks. In proceedings of the international conference on embedded networked sensor systems 2003 (pp. 17180). ACM.

[11] Lin P, Qiao C, Wang X. Medium access control with a dynamic duty cycle for sensor networks. In wireless communications and networking conference 2004 (pp. 1534-9). IEEE.

[12] Roy A, Sarma N. AEEMAC: adaptive energy efficient MAC protocol for wireless sensor networks. In India conference 2011 (pp. 1-6). IEEE.

[13] Boulfekhar S, Benmohammed M. Synchronous receiver initiated MAC protocol for long-lived sensor networks. Computers \& Electrical Engineering. 2014; 40(2):504-16.

[14] Polastre J, Hill J, Culler D. Versatile low power media access for wireless sensor networks. In proceedings of the international conference on embedded networked sensor systems 2004 (pp. 95-107). ACM.

[15] Buettner M, Yee GV, Anderson E, Han R. X-MAC: a short preamble MAC protocol for duty-cycled wireless sensor networks. In proceedings of the international conference on embedded networked sensor systems 2006 (pp. 307-20). ACM.

[16] Liu S, Fan KW, Sinha P. CMAC: an energy-efficient MAC layer protocol using convergent packet forwarding for wireless sensor networks. ACM Transactions on Sensor Networks. 2009; 5(4).

[17] Sun Y, Gurewitz O, Johnson DB. RI-MAC: a receiver-initiated asynchronous duty cycle MAC protocol for dynamic traffic loads in wireless sensor networks. In proceedings of the ACM conference on embedded network sensor systems 2008 (pp. 1-14). ACM.

[18] Tang L, Sun Y, Gurewitz O, Johnson DB. PW-MAC: an energy-efficient predictive-wakeup MAC protocol for wireless sensor networks. In INFOCOM proceedings 2011 (pp. 1305-13). IEEE.

[19] Bai RG, Qu YG, Guo Y, Zhao BH. An energyefficient TDMA MAC for wireless sensor networks. In Asia-pacific service computing conference 2007 (pp. 69-74). IEEE.

[20] Wang W, Wang H, Peng D, Sharif H. An energy efficient pre-schedule scheme for hybrid CSMA/TDMA MAC in wireless sensor networks. In Singapore international conference on communication systems 2006 (pp. 1-5). IEEE.

[21] Lee WL, Datta A, Cardell-Oliver R. FlexiMAC: a flexible TDMA-based MAC protocol for fault-tolerant and energy-efficient wireless sensor networks. In international conference on networks 2006 (pp. 1-6). IEEE.

[22] Handy MJ, Haase M, Timmermann D. Low energy adaptive clustering hierarchy with deterministic cluster-head selection. In international workshop on mobile and wireless communications network 2002 (pp. 368-72). IEEE.

[23] Ben-Othman J, Mokdad L, Yahya B. An energy efficient priority-based QoS MAC protocol for wireless sensor networks. In international conference on communications 2011 (pp. 1-6). IEEE.

[24] Lu G, Krishnamachari B, Raghavendra CS. An adaptive energy-efficient and low-latency MAC for data gathering in wireless sensor networks. In proceedings of international symposium of parallel and distributed processing 2004 (p. 224). IEEE.

[25] Ergen SC, Varaiya P. PEDAMACS: power efficient and delay aware medium access protocol for sensor networks. IEEE Transactions on Mobile Computing. 2006; 5(7):920-30.

[26] Cho S, Kanuri K, Cho JW, Lee JY, June SD. Dynamic energy efficient TDMA-based mac protocol for wireless sensor networks. In joint international conference on autonomic and autonomous systems and international conference on networking and services 2005 (pp. 1-5). IEEE. 
[27] Rajendran V, Obraczka K, Garcia-Luna-Aceves JJ. Energy-efficient, collision-free medium access control for wireless sensor networks. Wireless Networks. 2006; 12(1):63-78.

[28] Bao L, Garcia-Luna-Aceves JJ. A new approach to channel access scheduling for ad hoc networks. In proceedings of the annual international conference on mobile computing and networking 2001 (pp. 210-21). ACM.

[29] Pei G, Chien C. Low power TDMA in large wireless sensor networks. In military communications conference communications for network-centric operations: creating the information force 2001 (pp. 347-51). IEEE.

[30] Sohrabi K, Gao J, Ailawadhi V, Pottie GJ. Protocols for self-organization of a wireless sensor network. IEEE Personal Communications. 2000; 7(5):16-27.

[31] Van Hoesel L, Havinga P. A lightweight medium access protocol (LMAC) for wireless sensor networks. In international workshop on networked sensing systems 2004.

[32] Rashid RA, Embong WM, Zaharim A, Fisal N. Development of energy aware TDMA-based MAC protocol for wireless sensor network system. European Journal of Scientific Research. 2009; 30(4):571-8.

[33] Rhee I, Warrier A, Aia M, Min J, Sichitiu ML. ZMAC: a hybrid MAC for wireless sensor networks. IEEE/ACM Transactions on Networking. 2008; 16(3):511-24.

[34] Ahn GS, Hong SG, Miluzzo E, Campbell AT, Cuomo F. Funneling-MAC: a localized, sink-oriented MAC for boosting fidelity in sensor networks. In proceedings of the international conference on embedded networked sensor systems 2006 (pp. 293306). ACM.

[35] Liu Y, Ni LM. A new MAC protocol design for longterm applications in wireless sensor networks. In international conference on parallel and distributed systems 2007 (pp. 1-8). IEEE.

[36] Halkes GP, Langendoen KG. Crankshaft: an energyefficient MAC-protocol for dense wireless sensor networks. In European conference on wireless sensor networks 2007 (pp. 228-44). Springer, Berlin, Heidelberg.

[37] Gutierrez JA, Callaway EH, Barrett RL. Low-rate wireless personal area networks: enabling wireless sensors with IEEE 802.15. 4. IEEE Standards Association; 2004.

[38] Rhee I, Warrier A, Min J, Xu L. DRAND: distributed randomized TDMA scheduling for wireless ad hoc networks. IEEE Transactions on Mobile Computing. 2009; 8(10):1384-96.

[39] Ye W, Silva F, Heidemann J. Ultra-low duty cycle MAC with scheduled channel polling. In proceedings of the international conference on embedded networked sensor systems 2006 (pp. 321-34). ACM.

[40] Vural S, Tian Y, Ekici E. QoS-based communication protocols in wireless sensor networks. Algorithms and Protocols for Wireless Sensor Networks. 2009:365-99.

[41] Yigitel MA, Incel OD, Ersoy C. Design and implementation of a QoS-aware MAC protocol for wireless multimedia sensor networks. Computer Communications. 2011; 34(16):1991-2001.

[42] Liu Y, Elhanany I, Qi H. An energy-efficient QoSaware media access control protocol for wireless sensor networks. In international conference on mobile adhoc and sensor systems conference 2005 (pp. 1-3). IEEE.

[43] Saxena N, Roy A, Shin J. Dynamic duty cycle and adaptive contention window based QoS-MAC protocol for wireless multimedia sensor networks. Computer Networks. 2008; 52(13):2532-42.

[44] Paek KJ, Kim J, Song US, Hwang CS. Priority-based medium access control protocol for providing QoS in wireless sensor networks. IEICE Transactions on Information and Systems. 2007; 90(9):1448-51.

[45] Sitanayah L, Sreenan CJ, Brown KN. A hybrid MAC protocol for emergency response wireless sensor networks. Ad Hoc Networks. 2014; 20:77-95.

[46] Yu Q, Li G, Hang X, Fu K, Li T. An energy efficient MAC protocol for wireless passive sensor networks. Future Internet. 2017; 9(2):1-12.

[47] Zareei M, Islam AM, Vargas-Rosales C, Mansoor N, Goudarzi S, Rehmani MH. Mobility-aware medium access control protocols for wireless sensor networks: a survey. Journal of Network and Computer Applications. 2017; 104:21-37.

[48] Ait Aoudia F, Gautier M, Magno M, Berder O, Benini L. Leveraging energy harvesting and wake-up receivers for long-term wireless sensor networks. Sensors. 2018; 18(5):1-27.

[49] Ould Amara S, Beghdad R, Oussalah M. Securing wireless sensor networks: a survey. EDPACS. 2013; 47(2):6-29.

[50] Cheikhrouhou O. Secure group communication in wireless sensor networks: a survey. Journal of Network and Computer Applications. 2016; 61:11532.

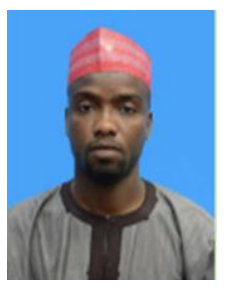

Bashir A Muzakkari is a lecturer in the Department of Computer Science, Northwest University, Kano, Nigeria. $\mathrm{He}$ is a graduate in Mobile Computing from Limkokwing University of Creative Technology, Malaysia. He is currently pursuing his $\mathrm{PhD}$ in Computer Science at Universiti Sultan

Zainal Abidin, Terengganu, Malaysia.

Email: muzakkar@gmail.com

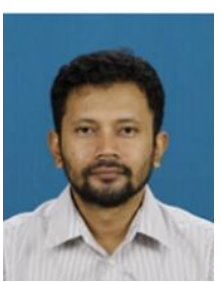

Networking

Email: mafendee@unisza.edu.my
Mohamad Afendee Mohamed received his $\mathrm{PhD}$ in Mathematical Cryptography and currently serves as a Lecturer at the Universiti Sultan Zainal Abidin. His research interests include both theoretical and application issues within the domains of Information Security, and Mobile and Wireless 
Muzakkari et al.

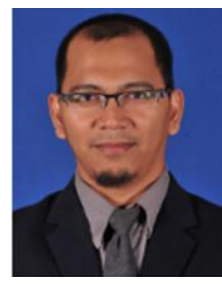

Mohd Fadzil Abdul Kadir received the B.Eng. degree (with honors) in Electrical and Electronic Engineering from the Mie University, Mie, Japan, in 2000, the M.Sc. degree in Information Technology from the Universiti Utara Malaysia (UUM), in 2004, and the $\mathrm{Ph} . \mathrm{D}$. degree in Engineering (System Engineering) from the Mie University, Mie, Japan, in 2012. Since 2006, he has been with the Faculty of Informatics \& Computing, Universiti Sultan Zainal Abidin (UniSZA), where he is currently a Senior Lecturer. His main areas of research interest are Digital Image Processing, Pattern Recognition, Computer Security and Cryptography. He is also a member of the Malaysia Board of Technologists.

Email: fadzil@unisza.edu.my

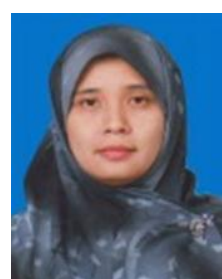

M. Zarina is an Associate Professor in the Faculty of Informatics and Computing, Universiti Sultan Zainal Abidin, Besut Campus, Terengganu, Malaysia. She holds a BSc from the Universiti Putra Malaysia and Ph.D from the Universiti Tun Hussien Onn Malaysia. Her research interests are Distributed Systems, Cloud Computing Management and Big Data.

Email: zarina@unisza.edu.my

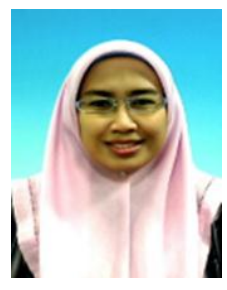

Norziana Jamil is an academic in Institute of Informatics and Computing in Energy, Universiti Tenaga Nasional. She received her $\mathrm{BSc}$ in Computer Science, MSc in Information Security and $\mathrm{PhD}$ in Security in Computing. Her area of specialization is Cryptography, Data Security, Security for Critical Infrastructure, Security of Internet of Things, Analytic for Data Protection and Smart City. She is a Program Manager for Uniten Smart UniverCity.

Email: norziana@uniten.edu.my 